\title{
Resiliencia en el confinamiento por el rebrote de COVID-19 en la comunidad San José
}

\author{
Resilience in confinement due to the outbreak of COVID-19 in the San José I community \\ Resiliência em confinamento devido ao surto de COVID-19 na comunidade San José \\ Luisa Celestina Jiménez Cedeño \\ luisajimenezcedeno@gmail.com \\ https://orcid.org/0000-0002-8575-667X \\ Leivi Jamilet Villamar Palacios \\ leivijvp94@gmail.com \\ https://orcid.org/0000-0001-8812-411X
}

Universidad Técnica de Manabí, Portoviejo, Ecuador

Inger Maitta

ingermaitta7@gmail.com

https://orcid.org/0000-0002-8695-5208

Universidad Técnica de Manabí, Portoviejo, Ecuador
Universidad Técnica de Manabí, Portoviejo, Ecuador

\section{RESUMEN}

El objetivo principal de esta investigación fue determinar los niveles de resiliencia en el confinamiento por el rebrote de COVID - 19 (Diciembre 2020- Enero 2021), en la comunidad San José I de la parroquia San Plácido de Ecuador. Se llevó a cabo la metodología descriptiva con un enfoque cualicuatitativo y el método inductivo para demostrar los niveles de resiliencia en el Confinamiento por el COVID-19 en esta comunidad. Para la recopilación de información se basó en una revisión bibliográfica y como instrumentos de medición se utilizó la Escala de Resiliencia SV-RES de Saavedra y una encuesta estructurada. Se concluyó que el confinamiento por el rebrote de COVID-19 no ejerce afectación en la resiliencia de las personas de la comunidad San José I de la parroquia San Plácido, esto debido a que las personas evaluadas presentan altos niveles de resiliencia, en su mayoría puntuando rangos medios y altos.

Palabras clave: Niveles de resiliencia; confinamiento; rebrote de COVID-19; comunidad;
ABSTRACT

The main objective of this research was to determine the levels of resilience in the confinement due to the outbreak of COVID - 19 (December 2020- January 2021), in the San José I community of the San Plácido parish of Ecuador. The descriptive methodology was carried out with a qualitativequalitative approach and the inductive method to demonstrate the levels of resilience in Confinement by COVID-19 in this community. Information gathering was based on a bibliographic review and the Saavedra Resilience Scale SV-RES and a structured survey were used as measurement instruments. It was concluded that the confinement due to the re-outbreak of COVID-19 does not affect the resilience of the people of the San José I community of the San Plácido parish, this because the people evaluated have high levels of resilience, mostly scoring middle and high ranges.

Key words: Levels of resilience; lockdown; outbreak of COVID-19; community; impact
O objetivo principal desta pesquisa foi determinar os níveis de resiliência no confinamento devido ao surto de COVID - 19 (dezembro de 2020 janeiro de 2021), na comunidade San José I da paróquia San Plácido do Equador. A metodologia descritiva foi realizada com abordagem qualitativo-qualitativa e o método indutivo para demonstrar os níveis de resiliência em Confinamento pelo COVID-19 nesta comunidade. A coleta de informações foi baseada em uma revisão bibliográfica e a Escala de Resiliência Saavedra SV-RES e um questionário estruturado foram usados como instrumentos de medida. Concluiuse que o confinamento devido ao ressurgimento do COVID-19 não afeta a resiliência da população da comunidade San José I da freguesia de San Plácido, isto porque as pessoas avaliadas apresentam elevados níveis de resiliência, principalmente com pontuação média e faixas altas.

Palavras-chave:

Níveis de resiliência; confinamento; surto de COVID-19; comunidade; impacto 


\section{INTRODUCCION}

Por la presencia del COVID-19, el mundo entero atraviesa una situación sanitaria que dejó como resultado consecuencias devastadoras con el contagio de la población y muerte de algunos de los que han adquirido este virus, colapsando el sistema de salud pública y con esta el estado emocional de las personas. Esta pandemia denominada COVID-19, ha generado en los individuos una ola de afectaciones a nivel psíquico, a lo que también están expuestos los habitantes de la comunidad San José I de la Parroquia San Plácido por el contagio ante el rebrote de este virus.

En tal sentido, es necesario tomar en cuenta que varias de estas personas requieren llevar un control de salud mental, debido a que optan en primera instancia de salvaguardar su integridad física (salvar su vida) dejando de lado las afectaciones psicológicas que se apoderan de las personas de la comunidad ante el confinamiento.

Durante el confinamiento, los dos factores que más afecta al bienestar físico y psicológico son la pérdida de hábitos y rutinas y el estrés psicosocial, de acuerdo al primer estudio que analiza el impacto psicológico de la cuarentena por COVID-19 en China (Wang, Pan et al., 2020).

Sin embargo, la resolución exitosa ante la adversidad presentada depende del nivel de resiliencia que tenga cada uno de los habitantes de la comunidad San José I, lo que permite disminuir el estrés, malestar, ansiedad y diversas alteraciones provocadas por el COVID-19, para así poder mejorar y elevar la calidad de vida. De la misma manera se consideró los efectos y las estrategias de afrontamiento que han surgido en los miembros de la comunidad San José I de la parroquia San Plácido a través de la experiencia durante el confinamiento por el rebrote del COVID-19.

Ante lo precedente, se planteó como objetivo general de investigación: Determinar los niveles de resiliencia en el confinamiento por el rebrote de COVID - 19 (Diciembre 2020- Enero 2021), en la comunidad San José I de la parroquia San Plácido.

Como objetivos específicos se propusieron los siguientes:

- Identificar los factores resilientes en el confinamiento por el rebrote de COVID- 19 (Diciembre 2020 - Enero 2021), en la comunidad San José I de la parroquia San Plácido.

- Establecer los efectos del confinamiento por el rebrote de COVID- 19 (Diciembre 2020 Enero 2021), en la comunidad San José I de la parroquia San Plácido.

- Describir las estrategias resilientes de los habitantes de la comunidad San José I de la parroquia San Plácido, en el confinamiento por el rebrote de COVID-19 (Diciembre 2020 Enero 2021).

Se justifica el presente estudio, entre diversas razones de impacto contextual, pues, ante esta compleja realidad, a través del estudio de investigación esta investigación se pudo abordar la relevancia de acciones psicológicas para mejorar la condición de vida de estos habitantes. Es necesario tener en cuenta, que el COVID-19 ha generado afectaciones de diferentes aspectos, la humanidad se encuentra golpeada y limitada por una pandemia que ha cambiado el estilo de vida de muchos, frenando o mutilando los sueños y aspiraciones de la gran mayoría de los seres humanos; pero que con el profesionalismo en el tratamiento psicológico y los altos niveles de resiliencia se logrará superar el estancamiento y salir victorioso de esta tragedia provocada por el COVID-19.

\section{COVID- 19 pandemia global}

A lo largo de la historia el mundo ha atravesado por varias pandemias y con estas el 
impacto negativo ha estado muy marcado debido a consecuencias devastadoras. Actualmente el rebrote del COVID-19 está presente en muchos países, los cuales se han visto perjudicados desde distintos aspectos de la salud física, salud mental, economía, educación, entre otros.

En el mes de diciembre de 2019, un brote de casos de una neumonía grave se inició en la ciudad de Wuhan, provincia de Hubei, en China. Los estudios epidemiológicos iniciales mostraron que la enfermedad se expandía rápidamente, que se comportaba más agresivamente en adultos entre los 30 y 79 años, con una letalidad global del 2,3\% (Wu \& McGoogan, 2020).

Esta enfermedad o síndrome respiratorio agudo severo tipo-2 (SARS-CoV-2) es el causante del COVID -19 y pertenece a la familia Coronavirus, dividiéndose en: Alphacoronavirus, Betacoronavirus, Gammacoronavirus y Deltacoronavirus, algunos de estos son causantes de enfermedades en animales domésticos.

Los coronavirus son una amplia familia de virus, algunos tienen la capacidad de transmitirse de los animales a las personas. Producen cuadros clínicos que van desde el resfriado común hasta enfermedades más graves, como ocurre con el coronavirus que causó el síndrome respiratorio agudo grave (SARS-CoV) y el coronavirus causante del síndrome respiratorio de Oriente Medio (MERS-CoV)". (MSP, 2020).

Este virus tiene un alto potencial de contagio y se transmite de forma rápida por medio de la tos, secreciones respiratorias de más de 5 micras que se pueden transmitir hasta los 2 metros de distancia, de la misma manera se produce cuando se tiene contacto con manos contaminadas, es decir, el contagio se da por contacto directo y cercano.
El 11 de marzo de 2020, la Organización Mundial de la Salud (OMS) declaró la pandemia de COVID-19, exhortando a todos los países a tomar medidas y aunar esfuerzos de control en lo que parece ser la mayor emergencia en la salud pública mundial de los tiempos modernos" (Adhanom, 2020 citado en Díaz \% Toro, 2020).

A raíz de esto Ecuador optó por medidas de bioseguridad como el cierre de varios servicios públicos, suspensión de labores presenciales, toque de queda, confinamiento, etc. Se marcaron medidas que mantuvieran distanciados unos de otros para evitar la propagación del contagio, lo que en su mayoría no fue así, sin embargo se produjo el cambio de todo el sistema nacional y el estilo de vida de las personas.

\section{Rebrote de Covid-19}

El término rebrote consiste en un nuevo brote, en este caso uno más fuerte y contagioso de COVID-19 que se ha presentado en Ecuador estos últimos meses debido a tres factores: el tiempo, el espacio y el número de personas que pueden estar involucradas. Haciendo referencia sobre las festividades y el clima, en diciembre del 2020 el Comité de Operaciones de Emergencia Nacional (COE), de Ecuador, pidió a las 221 alcaldías del país suspender eventos públicos y privados, ante el aumento de contagios por COVID-19. Es casi imposible frenar las actividades de la vida diaria, especialmente cuando los recursos son escasos y afectan la economía, en la gran mayoría de familias quien sale para buscar el sustento diario es el jefe de hogar, exponiéndose al contagio del virus y exponiendo a toda su familia, se hizo referencia al espacio o ambiente en el que el individuo se desenvuelve. Por otro lado al hablar del número de personas involucradas nos referimos al descontrol de estos, especialmente los jóvenes al no cumplir las normas de bioseguridad impuestas para la 
disminución del contagio. En Ecuador las ciudades con mayor cantidad de personas aglomeradas son Quito, Guayaquil, Cuenca y Portoviejo, aun cuando se exponen al peligro la sociedad no respeta el distanciamiento o las normas de bioseguridad.

Es evidente que la sociedad se encuentra afectada por esta situación, especialmente cuando las preocupaciones de un rebrote afectan la salud mental dado que la población se encuentra afectada al querer recuperar la economía del país tratando de evitar el rebrote de contagios y no se llegue a las mismas consecuencias que en China donde inició el rebrote en Xinjiang.

El COVID-19 se ha propagado en gran magnitud pero a diferencia de los primeros meses de la llegada de este virus, es menos letal, los casos graves y la mortalidad son bajos, debido a que al inicio afectó a la población más vulnerable, además porque el sistema sanitario no contaba con los recursos necesarios para enfrentar una crisis sanitaria.

\section{Confinamiento frente al COVID - 19}

Desde que el COVID - 19 se desató a nivel mundial, todos los países o la mayoría de ellos habían declarado estado de excepción, exigiendo normas que ayuden a la erradicar el contagio de esta cepa, Ecuador por su parte también declaró estas normas, una de ellas fue el confinamiento. Según la Real Academia Española (RAE) mencionan que el confinamiento es el "Aislamiento temporal y generalmente impuesto de una población, una persona o un grupo por razones de salud o de seguridad". El Gobierno de Ecuador tras la llegada del virus SARS-CoV-2 (COVID-19) lo impuso para toda la ciudadanía por primera vez el 12 de marzo de 2020 como una de las medidas de seguridad por la emergencia sanitaria.

"El confinamiento es un plan de intervención comunitario que implica permanecer refugiado el mayor tiempo posible" (Sánchez y De La Fuente,
2020). Junto a esto se toman y combinan estrategias que segreguen a las personas para no exponerse al contagio, además del confinamiento se utilizan métodos como el distanciamiento social, el uso de mascarillas, suspensión de eventos, restricción vehicular, entre otras, pero así como se toman medidas para la seguridad ciudadana se descuidan otras, el ser humano al ser un ente social no puede estar por mucho tiempo sin contacto con su medio, esto provoca que empiece, en ciertos casos, a experimentar las reacciones producto del aislamiento.

\section{Efectos del confinamiento}

Si bien la pandemia ha perjudicado la vida de las personas, el confinamiento que se ha tomado a raíz de esto ha provocado que en el mundo entero se presente un alto porcentaje de repercusiones a nivel financiero, social, físico, psicológico, emocional, etc. que se han manifestado de diversas formas en cada persona, "aparte de los efectos psicológicos negativos debidos directamente a las condiciones del confinamiento, las características de la propia pandemia y los múltiples factores asociados cualifican al confinamiento como una adversidad de elevado estrés psicosocial" (Sandin et al.,2020). Estas repercusiones generadas por el confinamiento y el COVID -19 impactan la salud mental de los individuos, que al encontrarse vulnerables ante esta crisis tienden a presentar problemas psicológicos y emocionales a niveles más altos.

Existen evidencias que determinan que el confinamiento deteriora la salud mental, "Las enfermedades mentales afectan a gran parte de la población durante y después de la cuarentena, existen los siguientes estresores, que son parte de esta experiencia y pueden generar estos cuadros: el periodo de duración de la cuarentena, el miedo de infectarse o infectar a otros, la frustración o aburrimiento debido al confinamiento o pérdida 
de rutina, así como la pérdida de contacto social y físico con otros". (Espinosa, et al, 2020). Esto quiere decir que el tiempo es de gran importancia para quienes se encuentran recluidos, mientras mayor sea su prolongación mayor será la afectación, de la misma manera, el miedo al contagio especialmente cuando son parte de los grupos vulnerables. Según Balluerca y otros autores (2020) refuerzan que:

los factores que más afectan al bienestar físico como el psicológico son, la pérdida de hábitos y rutinas, la instauración de otros poco saludables y el estrés psicosocial; de acuerdo con el primer estudio que analiza el impacto psicológico de la cuarentena por COVID-19 en China.

Como es bien sabido la parte psicológica es la que mayor afectación tiene durante el confinamiento, pero esta se entrelaza por problemas relacionados a otras áreas del individuo, como son:

Efectos económicos y/o laborales: Con el confinamiento la economía se paralizó, las más afectadas fueron las personas de escasos recursos, es decir, los más vulnerables, muchos quedaron sin empleo, por ende sin sueldo, debido al cierre de empresas o instituciones, y sin ahorros es complicado pasar una crisis con estas medidas. Aproximadamente hubo pérdidas de hasta $750 \mathrm{mil}$ empleos y la pobreza aumentó un $10 \%$.

Efectos psicosociales: "El estado emocional de un sujeto puede verse afectado por la exposición al sufrimiento, las quejas y otras expresiones emocionales de sus contactos sociales, lo que significa que el estado de ánimo y el estrés pueden propagarse de persona a persona, por un proceso llamado "contagio emocional". (Bartholomew \& Zaldivar, 2018). A causa del cambió de las actividades rutinarias con el confinamiento, por ejemplo la asistencia a las instituciones educativas y laborales, salir a fiestas, reunirse con amigos o colegas, etc, y ya que el ser humano es un ente social, las personas presentan mayor sensación de soledad, especialmente las de tercera edad, a pesar de que se utilizan diferentes medios para mantener la comunicación (WhatsApp, Facebook, Instagram , Zoom, etc.) la ansiedad ha reinado en este tiempo. La carencia de contacto social y la sensación de soledad y aislamiento son factores que afectan también en la salud mental de las personas.

Efectos familiares: Durante este tiempo de confinamiento las relaciones familiares se han desarrollado de la mejor manera, los vínculos afectivos se han fortalecidos, ya que juntos atraviesan la misma situación, sobrevivir a esta pandemia se ha convertido en un reto familiar. Aunque no en todos los casos ha sido igual, debido al estrés provocado por las nuevas responsabilidades del hogar, las intensas preocupaciones que rodean a la familia, y muchas más, estas se han visto en la obligación de desplegar nuevos modos de convivencia, al igual que se han desarrollado nuevos modos de violencia, ya sea en hogares donde no existía la misma o el incremento en familias que anteriormente presentaban una relación violenta. En Ecuador en el mes de abril del 2020 según registros del ECU 911 la violencia aumentó a un $47 \%$ y sigue creciendo el porcentaje, la mayor parte de estas llamadas fueron realizadas por mujeres que denunciaron casos de violencia en su hogar.

Efectos fisiológicos: Existen tres razones por las cuales se presenta este tipo de afectación: 1. Las personas vulnerables que han presentado síntomas del COVID - 19 u otras enfermedades. 2. Las personas que debido al confinamiento han desarrollado una pérdida de hábitos y rutinas, por ejemplo el sedentarismo, los malos hábitos alimenticios, abuso de horas frente a dispositivos electrónicos, inactividad física, etc., lo que ha causado 
consecuencias en el cuerpo; 3. Debido a factores psicológicos como el estrés y la ansiedad que provocan el aumento del ritmo cardiaco y/o tensión arterial, lo cual el mantenimiento de esta situación a largo plazo provoca consecuencias físicas de gran magnitud, estas afectaciones psicológicas que ya de por si provocan en el individuo alteraciones del sueño, alteraciones gastrointestinales, cambios hormonales, etc., también pueden afectar a nivel músculo -esquelético.

Efectos emocionales y psicológicos: Ciertas reacciones emocionales como estrés, ansiedad, agobio, miedo y ansiedad, incluso angustia o irritabilidad, son normales durante este tiempo debido a la situación que atraviesa el mundo entero por causa de la pandemia (COVID-19) y sus medidas preventivas, estos síntomas trabajan como mecanismos de defensa ante la crisis, "el conjunto de emociones negativas de miedo, ansiedad y preocupación suele darse de forma mixta en situaciones de elevado estrés psicológico y ante amenazas de naturaleza impredecible e incontrolable" (Sandín, 2009, citado en Abate, Urtecho\& Agüero, 2020), como la crisis y las medidas que se usan para salvaguardar la vida, pero que además se toman como causas de sintomatologías que perjudica la salud mental, provocando emociones como estrés, desesperación, nostalgia, alteraciones del sueño, alteraciones de atención y concentración, impotencia, agotamiento, frustración, irritabilidad, etc. De la misma manera pueden manifestarse o empeorar los trastornos mentales, la ansiedad, el estrés y la depresión son los que más se han desarrollados durante el confinamiento y en personas con diagnósticos psiquiátricos pueden agravar el estado de salud mental.

El trastorno adaptativo es otra de las afectaciones que por una parte se considera normal ante los estímulos estresores producidos por la pandemia, pero si sus síntomas no cesan en pocos meses pueden empeorarse, generando nuevos trastornos. "Los trastornos adaptativos se encuentran en el límite entre la normalidad y la patología mental, es decir, que son una especie de puente entre las personas que logran adaptarse a la nueva condición y las que se sienten sobrepasadas por lo que está aconteciendo y desarrollan trastornos psiquiátricos" (Muñoz, et. al, 2020). En muchas personas el consumo de alcohol y sustancias ha aumentado tomando esta acción como manera de afrontar los miedos que atraviesan por la pandemia, lo que causa que la ansiedad y la depresión incremente.

El trastorno de estrés postraumático (TEPT) es uno de los trastornos que se pueden presentar a futuro, provocando pensamientos intrusivos frecuentes, conductas de evitación, alteraciones cognitivas y del estado de ánimo, conductas impulsivas, irritabilidad, etc. De la misma manera los trastornos depresivos, el estrés agudo, trastornos psicosomáticos o conductas suicidas, pueden exteriorizarse durante esta época.

\section{Conceptos de resiliencia}

A lo largo del tiempo varios autores han estudiado la resiliencia y sus definiciones han evolucionado a medida que el conocimiento científico ha aumentado. Luthar (2000, citado en Ascencio, 2015) se refiere a un "proceso dinámico que abarca la adaptación positiva dentro del contexto de una adversidad significativa".

Osorio y Romero (2003, citado en Ascencio, 2015), refieren quees la“capacidad universal de todo ser humano de resistir ante condiciones adversas y recuperase, desarrollando paulatinamente respuestas orientadas hacia la construcción de un ajuste psicosocial positivo al entorno, a pesar de la existencia simultánea de dolor y conflicto intrapsíquico".

Richardson, Neieger, Jensen y Kumpfer (1990) definen la resiliencia como "el proceso de 
afrontamiento con eventos vitales desgarradores, estresantes o desafiantes de un modo que proporciona al individuo protección adicional y habilidades de afrontamiento que las que tenía previa a la ruptura que resultó desde el evento" (Ivorra, 2017), de la misma manera Quishpe (2019) da un concepto más actualizado, este autor menciona que "La resiliencia puede ser entendida como una medida de la capacidad para afrontar el suceso estresante, debido a que no solo se refiere a resistir la situación amenazante, sino a su vez mantener el equilibrio sin que afecte la vida diaria", se puede considerar a este concepto como semejante a la supervivencia, ya que la persona enfrenta sucesos muy difíciles y sale triunfante en la vida. La resiliencia se refiere a la adaptación positiva, o la capacidad de mantener o recuperar la salud mental, a pesar de haber experimentado adversidades, una persona resiliente no está exenta de presentar estrés, es decir no es invulnerable, sino lo que marca esta capacidad es la habilidad con que se recupera o afronta el evento negativo, así lo menciona Muñoz (2020).

Ser resiliente no significa ausencia de malestar, dolor emocional o dificultad ante las adversidades tales como la muerte de un ser querido, una enfermedad grave, la pérdida del trabajo, problemas socio económicos y otros que generan impacto, y que producen una sensación de inseguridad, incertidumbre y dolor emocional. Las personas logran, por lo general, sobreponerse a esas situaciones y adaptarse bien a lo largo del tiempo; el camino que lleva a la resiliencia implica enfrentarse a factores estresantes y a malestares emocionales; de esta forma las personas sacan la fuerza que les permiten seguir con sus vidas frente a la adversidad, lo cual implica una serie de conductas y formas de pensar que los seres humanos aprenden a desarrollar.

Existen varias definiciones sobre la resiliencia, algunas de estas marcan a la misma como un rasgo personal que opera después de un solo trauma de corta duración, pero la diferencia es que esta capacidad es una dinámica en proceso, debido a que puede variar a lo largo del tiempo, dependiendo las etapas y sucesos de la vida, manifestándose en conductas y patrones adaptativos. Según Masten (2018) "la resiliencia es una característica propia del desarrollo evolutivo y, por tanto, puede observarse en todas las personas en mayor o menor medida", comparándose con el desarrollo Darwiniano centrándose en lo selectivo, fortalezas o activos, adaptándose el sujeto al estímulo rodeado para aumentar la capacidad de aprendizaje y afrontamiento no solo de forma individual, sino que además de manera social y así transformarse al cambio global. Para Saavedra et al. (2015) "la resiliencia es dinámica y está en constante desarrollo, mediante la interacción entre los sujetos y su entorno, no es una condición absoluta". Por lo tanto, la resiliencia se refiere a la capacidad de un sistema para continuar en su estado actual de desempeño mientras enfrenta interrupciones y cambios, se adapta a los desafíos futuros y se transforma de manera que mejore su funcionamiento.

\section{Tipos de resiliencia}

\section{Resiliencia individual}

Es tomada como la principal entre los otros tipos de resiliencias, representa al potencial que el ser humano tiene para surgir victorioso ante experiencias adversas, complejas o difíciles, incluye comportamientos, pensamientos y acciones que promueven la salud mental, así como lo menciona Villegas (2017) "es la capacidad que posee un individuo de sobreponerse ante cualquier situación adversa. Para ello es necesario que posea unas determinadas características: Autoestima, humor, entusiasmo, seguridad, confianza, estrategias para resolver conflictos etc". Desarrollar la capacidad 
resiliente ayuda a que el individuo se adaptarse y recupere ante un estímulo estresor.

\section{Resiliencia familiar}

(Villegas, 2017) "Es la capacidad que tiene una familia de sobreponerse a cualquier adversidad, unidos y contando con el apoyo de los unos a los otros, para ello es necesario que haya una estabilidad y armonía familiar, se tengan en cuenta las opiniones de los individuos que la integran etc", fortalece al sistema para superar adversidades futuras como una unidad funcional, debido a que la familia se mueve como un sistema lo que le afecta a un individuo puede alterar todo el conjunto, ya que en ocasiones no afecta tanto a la familia el hecho traumático sino la representación que se tiene de este.

\section{Resiliencia social}

Este término hace referencia a la "la capacidad que tiene el entorno de superar los problemas, aprender de los errores etc., contando con el apoyo conjunto y la cooperación de los individuos que integran ese grupo social" (Villegas, 2017), mejora su estructura, fortaleciendo los recursos con los que la comunidad cuenta, influyen en esta las respuestas psicosociales así como las individuales. Este tipo de resiliencia se presenta ante impactos sociales como atentados, conflictos colectivos, terremotos, inundaciones, pandemias, de la misma manera una represión política y otras, que afectan a la sociedad en general.

\section{MÉTODO}

La presente investigación fue de tipo descriptiva ya que permitió llevar un registro, análisis e interpretación de la naturaleza actual y la composición o procesos de los fenómenos. Así mismo, presenta un enfoque mixto porque permite obtener la recolección de datos y análisis de manera cuantitativa y cualitativa.

En cuanto a la identificación de las variables se concretó lo siguiente:
Variable Independiente: Resiliencia
- Variable Dependiente: Confinamiento por rebrote de COVID-19

\section{RESULTADOS}

Para el desarrollo de esta investigación se contó con la aprobación del presidente de la comunidad San José I de la parroquia San Plácido, evaluando y obteniendo los resultados mediante la aplicación de la escala de resiliencia SV-RES y la encuesta estructurada, al comenzar la aplicación de dichos instrumentos se explicó a cada una de las personas el objetivo de la investigación.

Los primeros resultados se corresponden a la Escala de Resiliencia utilizada, y a continuación, se encuentra el análisis a los resultados obtenidos mediante la encuesta semiestructurada aplicada; finalmente, se halla la explicación por parte de las autoras de la investigación, contrastando los resultados de los instrumentos aplicados con anterioridad, dando de esta manera respuesta a los objetivos planteados inicialmente en el trabajo.

\section{Escala de Resiliencia SV-RES de Saavedra}

Objetivo específico: Identificar los niveles de resiliencia en el confinamiento por el rebrote de COVID- 19 (Diciembre 2020 - Enero 2021), en la comunidad San José I de la parroquia San Plácido. 
Dentro del proceso de investigación sobre resiliencia en el confinamiento por el rebrote de Covid-19 en la comunidad San José I de la parroquia San plácido, se utilizaron varios instrumentos para poder obtener información sobre la situación de la comunidad, uno de los instrumentos aplicados en este proceso fue el Test de Resiliencia SV-RES de Saavedra, en el que se obtuvieron los siguientes resultados (Tabla 1), el nivel de resiliencia alto fue de $53 \%$, mientras que el nivel de resiliencia medio fue $39 \%$, por lo consiguiente el nivel de resiliencia bajo fue de $8 \%$, lo que indica que los habitantes de esta comunidad en su gran mayoría tiene la capacidad de afrontar las adversidades que se presenten en la vida, a pesar de que seguimos con el confinamiento por el rebrote del COVID-19 no se muestran con actitudes negativas, lo que les permite avanzar en su diario vivir a pesar de las limitaciones por el confinamiento.

Tabla 1. Resultados de escala SV-RES de Saavedra.

\begin{tabular}{cc}
\hline Nivel de resiliencia & $\begin{array}{c}\text { Porcentaje de los niveles de resiliencia en la } \\
\text { comunidad San José I de la Parroquia "S.P." }\end{array}$ \\
\hline Alto & $53 \%$ \\
Medio & $39 \%$ \\
Bajo & $8 \%$ \\
\hline
\end{tabular}

Tabla 2. Factores de la Resiliencia.

\begin{tabular}{cccccc}
\hline Yo soy, yo estoy & Identidad & Autonomía & Satisfacción & Pragmatismo & Total \\
& $25 \%$ & $25 \%$ & $25 \%$ & $25 \%$ & $100 \%$ \\
Yo tengo & Vínculos & Redes & Modelo & Metas & Total \\
& $25 \%$ & $25 \%$ & $25 \%$ & $25 \%$ & $100 \%$ \\
Yo puedo & Afectividad & Autoeficacia & Aprendizaje & Generatividad & Total \\
& $25 \%$ & $25 \%$ & $25 \%$ & $25 \%$ & $100 \%$ \\
\hline
\end{tabular}

\section{Análisis e interpretación}

Por otro lado, se pueden observar los porcentajes de los 12 factores de resiliencia propuestos en el test SV-RES, en el que se abordan los diversos niveles que contribuyen a la formación de la resiliencia como el "Yo tengo" que corresponde al entorno social, el "Yo soy" y "Yo estoy" que se refiere a la fuerza psicológica interna del sujeto y el "Yo puedo" que correspondiente a las habilidades en las relaciones con los otros.

Dentro del nivel "yo soy, yo estoy", se observa en la tabla 2 que el factor de identidad que presenta el porcentaje con un $25 \%$, que habla sobre los juicios generales que el sujeto define sobre sus valores culturales de un modo estable, le sigue el pragmatismo con un $25 \%$, que refiere a la forma que el sujeto interpreta las acciones que realiza. La autonomía con un $25 \%$ que hace referencia a los juicios del vínculo del sujeto consigo mismo y su aporte con el entorno social y cultural y la satisfacción con un $25 \%$ que representa a las formas de interpretar las situaciones adversas.

En el nivel "Yo tengo" se distingue un porcentaje de $25 \%$ en vínculos que representa a los juicios del individuo sobre las redes sociales con raíces en su historia personal. También se destaca 
un puntaje medio con el $25 \%$ en redes que hace referencia a los juicios del individuo con respecto al vínculo afectivo que tiene con el entorno. Se menciona también un $25 \%$ en modelo que son los juicios del sujeto sobre la convicción de las redes sociales cercanas y su apoyo para la superación de situaciones adversas y se observa también un $25 \%$ en metas que son los juicios del valor contextual de las metas y redes sociales sobre la situación problema.

En el nivel "Yo puedo" se observa un $25 \%$ en afectividad que refiere al juicio de las posibilidades sobre sí mismo y su vínculo con los demás. También se evidencia el $25 \%$ en autoeficacia que refiere a las posibilidades de éxito que las personas reconocen de sí mismo ante los problemas, también se obtuvo el $25 \%$ en aprendizaje que se refiere al valor de las personas sobre las situaciones adversas y un posible aprendizaje y un $25 \%$ en generatividad que representa a la posibilidad que los habitantes de la comunidad identifican para pedir ayuda a otras personas en situaciones problemáticas.

\section{Resultados de escala SV-RES de Saavedra por dimensión}

Los resultados de los factores YO SOY, YO ESTOY de la Dimensión 1-identidad sobre la comunidad San José I de la parroquia san plácido indican los siguientes resultados, alcanza un mayor número de personas en promedio de nivel de resiliencia alto con un $60 \%$, mientras que un nivel de resiliencia medio con un $30 \%$, y por otra parte un nivel de resiliencia bajo con un $10 \%$.

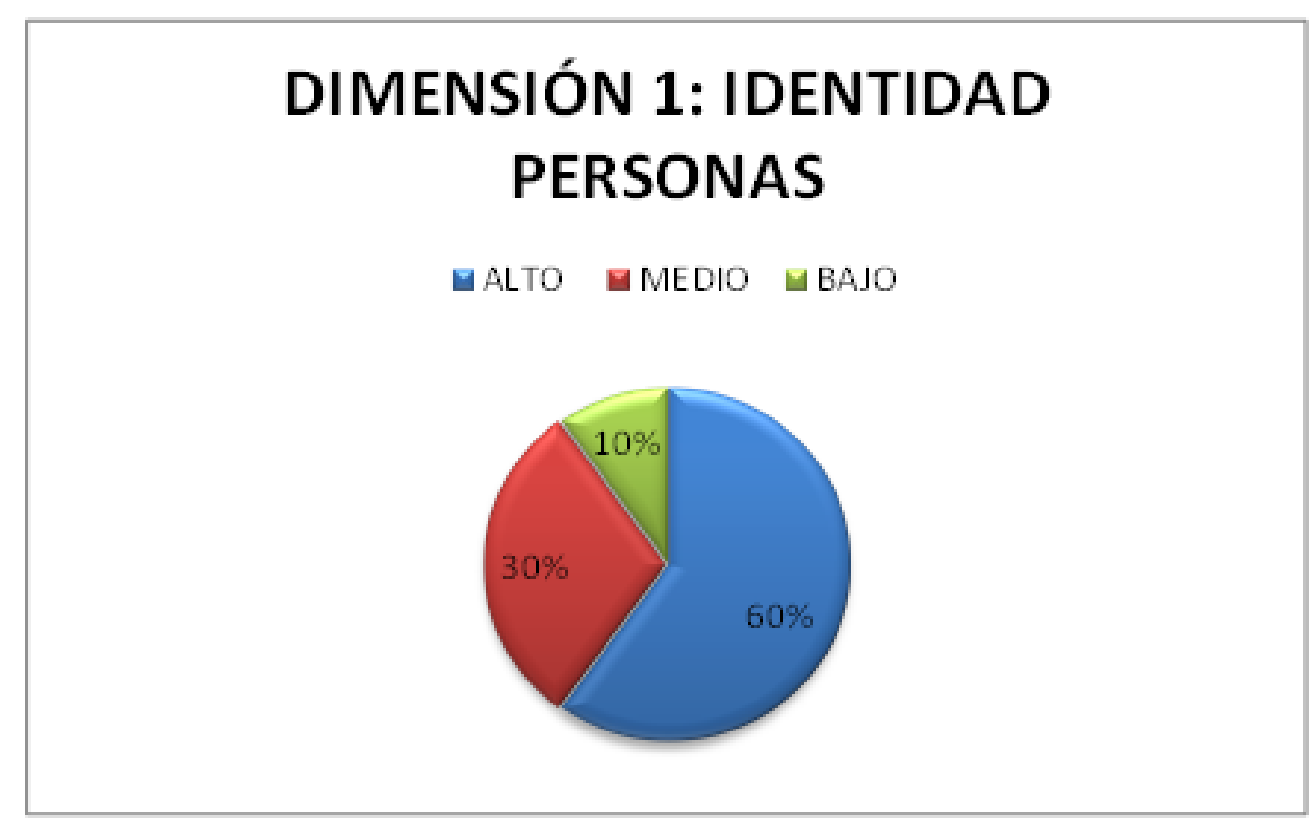

Gráfico 1. Dimensión Identidad (Fuente: Comunidad San José I de la parroquia San Plácido).

Los derivados de los factores YOSOY, YO ESTOY de la Dimensión 2-Autonomía sobre la comunidad San José I de la parroquia San Plácido indican los siguientes resultados, alcanza un mayor número de personas en promedio de nivel de resiliencia alto con un $56 \%$, mientras que un nivel de resiliencia medio con un $32 \%$, y por otra parte un nivel de resiliencia bajo con el $12 \%$. 


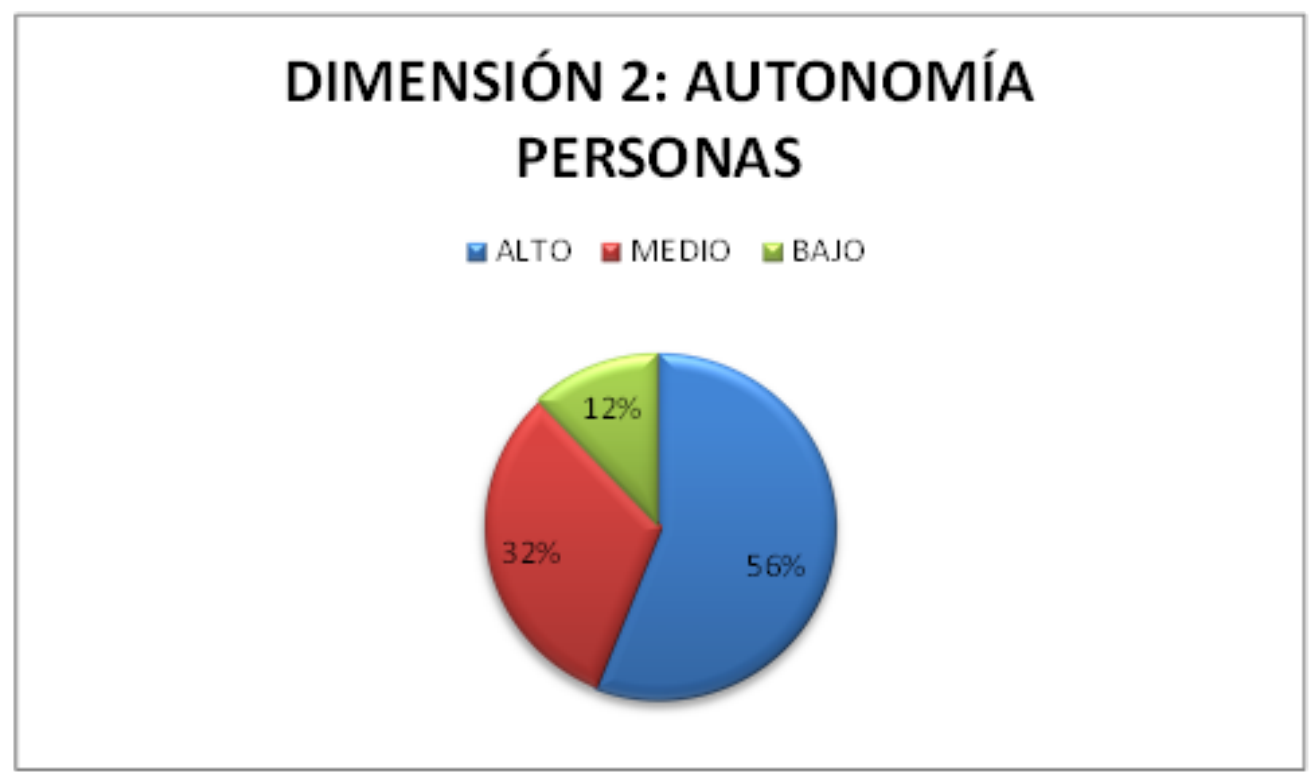

Gráfico 2. Dimensión Autonomía (Fuente: Comunidad San José I de la parroquia San Plácido).

Los resultados de los factores YO SOY, YO ESTOY de la Dimensión 3-Satisfacción sobre la comunidad San José I de la parroquia San Plácido indican los siguientes resultados, alcanza un mayor número de personas en promedio de nivel de resiliencia alto con un $62 \%$, mientras que un nivel de resiliencia medio con un $20 \%$, y por otra parte un nivel de resiliencia bajo con el $18 \%$.

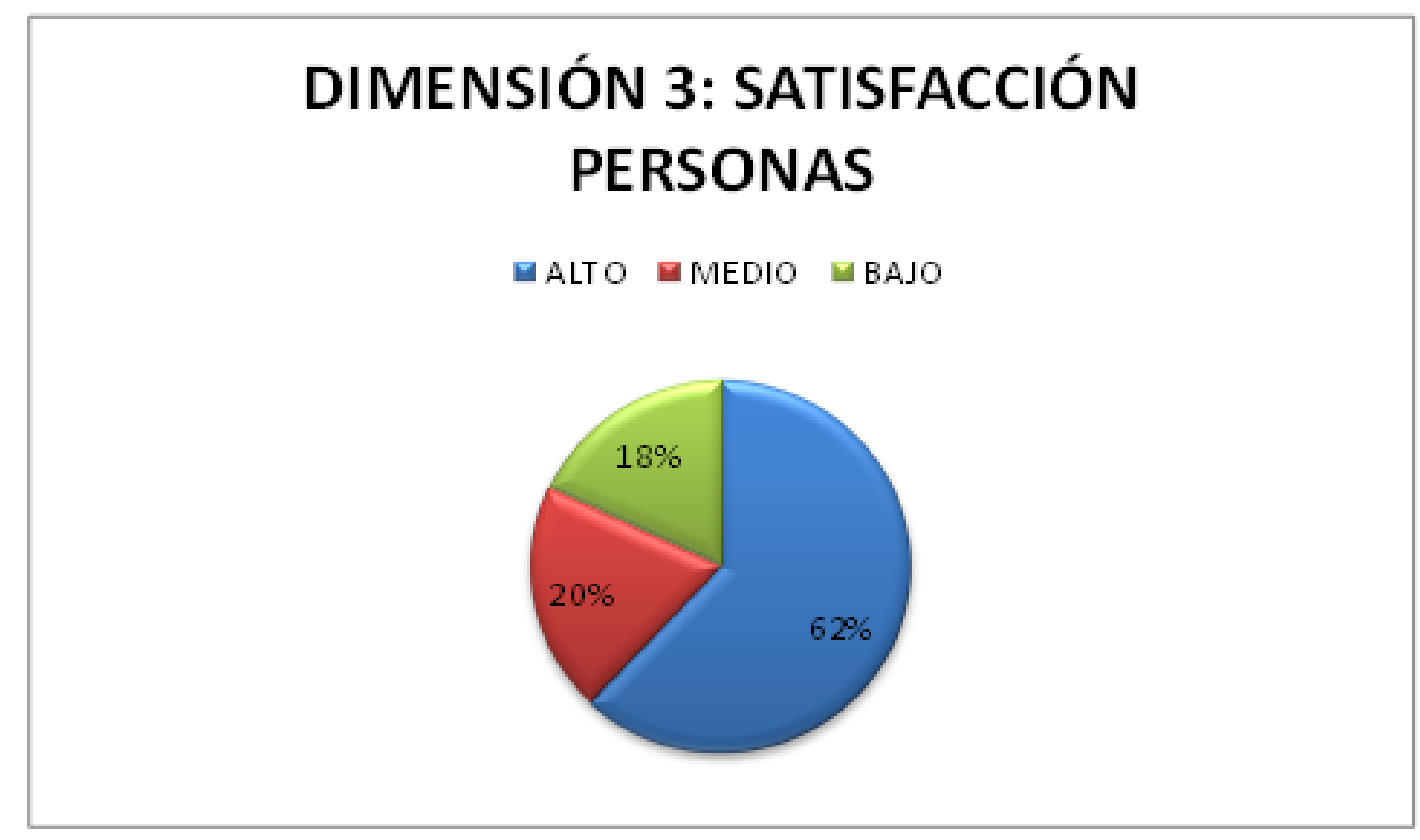

Gráfico 3. Dimensión satisfacción (Fuente: Comunidad San José I de la parroquia San Plácido). 
Los resultados de los factores YO SOY, YO ESTOY de la Dimensión 4-Pragmatismo sobre la comunidad San José I de la parroquia San Plácido indican los siguientes resultados, alcanza un mayor número de personas en promedio de nivel de resiliencia alto con el $66 \%$, mientras que un nivel de resiliencia medio con un $32 \%$, y por otra parte un nivel de resiliencia bajo con el $2 \%$.

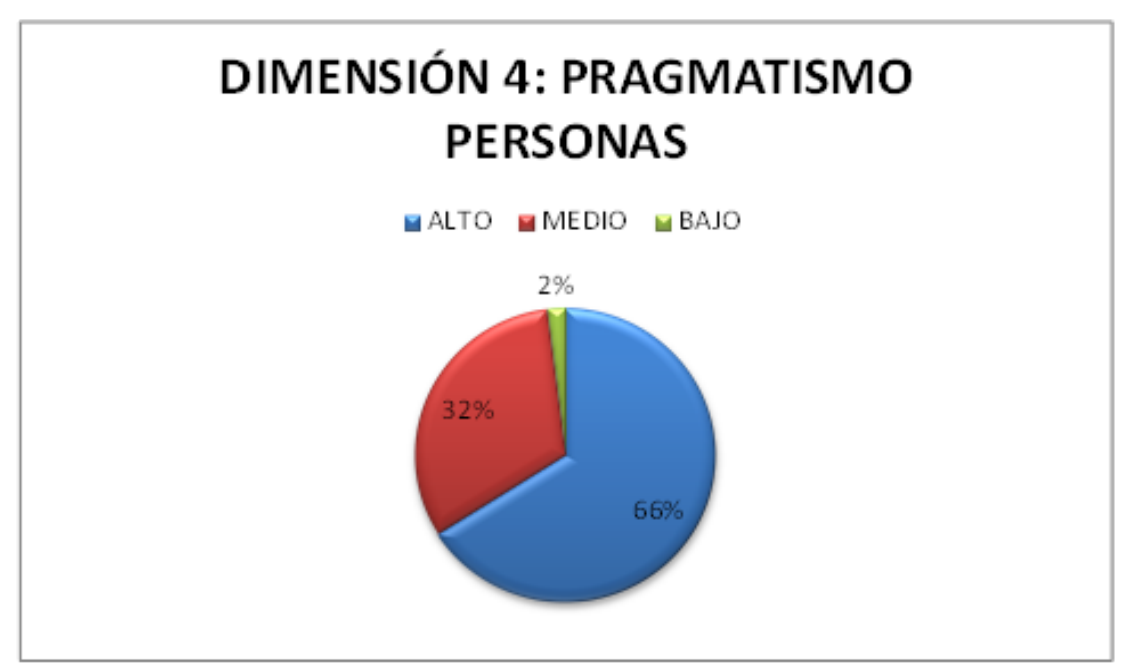

Gráfico 4. Dimensión Pragmatismo. (Fuente: Comunidad San José I de la parroquia San Plácido).

Los resultados de los factores YO TENGO de la dimensión 5-Vinculos sobre la comunidad San José I de la parroquia San Plácido indican los siguientes resultados, alcanza un promedio en personas de nivel de resiliencia alto con el $38 \%$, mientras que un nivel de resiliencia medio con un $52 \%$, y por otra parte un nivel de resiliencia bajo con el $10 \%$.

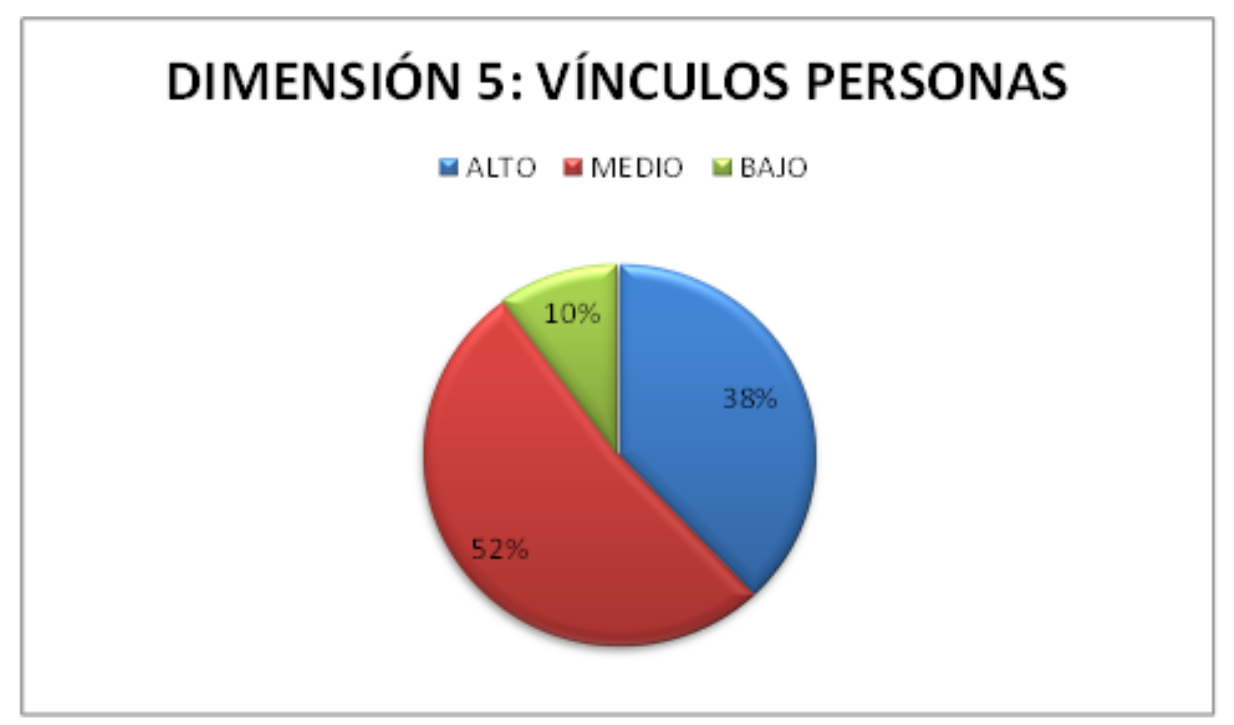

Gráfico 5. Dimensión Vínculos Personas. (Fuente: Comunidad San José I de la parroquia San Plácido). 
Se deduce de los factores YO TENGO de la dimensión 6-Redes sobre la comunidad San José I de la parroquia San Plácido indican los siguientes resultados: alcanza un promedio en personas de nivel de resiliencia alto con el $66 \%$, mientras que un nivel de resiliencia medio con un $24 \%$, y por otra parte un nivel de resiliencia bajo con el $10 \%$.

\section{DIMENSIÓN 6: REDES PERSONAS}

$\square$ ALTO $\square$ MEDIO $\square$ BAJO

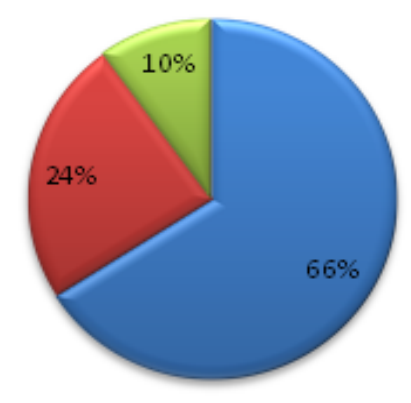

Gráfico 6. Dimensión Redes. (Fuente: Comunidad San José I de la parroquia San Plácido).

Lo derivado de los factores YO TENGO de la dimensión 7-Modelos sobre la comunidad San José I de la parroquia San Plácido indican los siguientes resultados, alcanza un promedio en personas de nivel de resiliencia alto con el $28 \%$, mientras que un nivel de resiliencia medio con el $64 \%$, y por otra parte un nivel de resiliencia bajo con el $8 \%$.

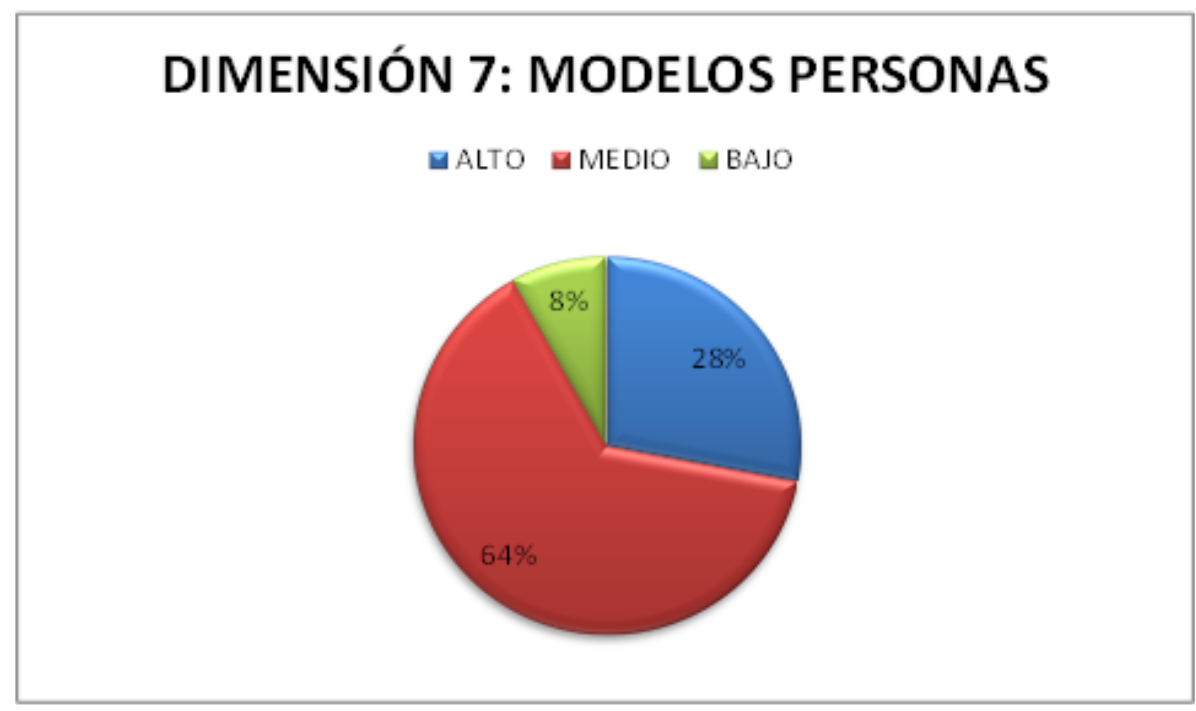

Gráfico 7. Dimensión Modelos Personas. (Fuente: Comunidad San José I de la parroquia San Plácido). 


\section{CONCLUSIONES}

Se pudo llegar a concluir que durante el período de confinamiento por el rebrote de COVID-19, los habitantes delacomunidadSan Joséldelaparroquia San Plácido presentaron varios efectos a nivel psicológico como miedo, nerviosismo, ansiedad, fatiga, etc, esto desencadenado especialmente por el impacto económico y laboral, así como el estado de la pandemia misma durante este periodo. A pesar de la afectación emocional que se produjo por el Confinamiento, los habitantes de dicha comunidad resaltan presentar buena salud mental, la misma que se ve reflejada en los resultados de los niveles de resiliencia obtenidos, logrando así manejar adecuadamente sus emociones haciendo que estos efectos no interfieran en su bienestar.

La preocupante situación que atraviesa el mundo entero ha llevado a los habitantes de la comunidad San José 1 de la parroquia San Plácido desarrollar alternativas saludables para poder soportar la situación y los efectos causados por la pandemia, Estos han aplicado consciente o inconscientemente estrategias de afrontamiento que permiten mantenerse conectados a la realidad sin que afecte su salud mental. La mayoría de estrategias desarrolladas involucran estilos de manejo de emociones, siendo expresivos y empáticos consigo mismos y con los demás, las prácticas religiosas o espirituales son otras que han tenido bastante peso durante este tiempo, de la misma manera los estilos que van directamente a la acción cómo realizar ejercicios físicos, buscar diversiones, planificar soluciones, entre otras, todo esto ha contribuido para que la población sea resiliente y se mantenga estable durante esta y posibles futuras adversidades.

\section{REFERENCIAS}

Abate, C. Urtecho, O. y Agüero, M. (: 23 noviembre 2020). Efectos psicológicos del confinamiento social por COVID-19 en la población hondureña. Revista Ciencia y Tecnología. Dirección de Investigación Científica, Humanística y Tecnológica. Universidad Nacional Autónoma de Honduras. DOI 10.5377/rct.v13i25.10409

Ascencio, A. (2015). Resiliencia y Estilos de Afrontamiento en mujeres con Cáncer de mama de un Hospital Estatal de Chiclayo 2013. Universidad Católica Santo Toribio de Mogrovejo. Chiclayo. https://core.ac.uk/ download/pdf/71999801.pdf

Balluerca, N., Gómez, J., Hidalgo, M., Gorostiaga, A., Espada, J, Padilla, J y Santed, M. (2.020). Las consecuencias psicológicas de la COVID-19 y el confinamiento. (Informe de Investigación). Servicio de Publicaciones de la Universidad del País Vasco.

Bartholomew, R.yZaldivar, D.(2018). Chasngghost in Cuba; Is Mass psychogenic illness masquerading as an acoustical at-tack? International Journal of Social Psychiatry, 64(5), 413-416. https://doi. org/10.1177\%2F0020764018766185

Espinosa Y. Mesa D. Diaz Y. Caraballo L. Mesa M. (2020) Estudio del impacto psicológico de la COVID-19 en estudiantes de Ciencias Médicas, Los Palacios. Revista Cubana de Salud Pública. http://scielo.sld.cu/pdf/rcsp/v46s1/1561-3127rcsp-46-s1-e2659.pdf

Ivorra, B. (2017). Factores amortiguadores del impacto de la enfermedad como predictores de resiliencia en la esclerosis múltiple. Universidad Miguel Hernández de Elche. http://dspace.umh. es/bitstream/11000/4541/1/TD\%20lvorra\%20 Navarro\%2C\%20B\%C3\%A1rbara.pdf

López, A., y Vásquez, L. (2020). LA RESILIENCIA COMO UN PRINCIPIO DE VIDA A TRAVÉS DE LA COVID-19.Creativecommons. https://repository. ucatolica.edu.co/bitstream/10983/24968/1/ TRABAJO\%20FINAL\%20DE\%20GRADO.pdf 
Masten, A. S. (2018). Resilience theory and research on children and families: Past, present, and promise. Journal of Family Theory y Review, 10, 12-31. https://onlinelibrary.wiley.com/doi/ abs/10.1111/jftr.12255

MSP. (Abril de 2020). Coronavirus COVID-19. https:// www.salud.gob.ec/coronavirus-covid-19/

Muñoz, S., Molina, D., Ochoa, R., Sánchez, O., y Esquivel, J. (2020) Estrés, respuestas emocionales, factores de riesgo, psicopatología y manejo del personal de salud durante la pandemia por COVID-19. Acta Pediatr Méx 2020; 41 (Supl 1):S127-S136. https://www. medigraphic.com/pdfs/actpedmex/apm-2020/ apms201q.pdf

Saavedra, E., Salas, G., Cornejo, C., y Morales, P. (2015). Resiliencia y Calidad de Vida: la Psicología Educacional en diálogo con otras disciplinas. Talca: UCM.

Sánchez-Villena AR y De La Fuente- Figuerola V., (2.020). COVID-19: cuarentena, aislamiento, distanciamiento social y confinamiento, ¿son lo mismo? An Pediatr (Barc). https://doi. org/10.1016/j.anpedi.2020.05.001

Sandín, B., Valiente, R. M., García-Escalera, J., y Chorot, P. (2020). Impacto psicológico de la pandemia de COVID-19: Efectos negativos y positivos en población española asociados al periodo de confinamiento nacional. Revista de Psicopatología y Psicología Clínica, 25(1), 1-11

Vargas, J., y Montalvo, A. (2017). "PROCESO DE RESILIENCIA PARA LA SUPERACIÓN DE RIESGOS SOCIALES EN LOS NIÑOS, NIÑAS Y ADOLESCENTES DE LA ONG GEMA-HUANCAYO 1990-2016". Universidad Nacional Del Centro Del Perú. http://repositorio.uncp.edu.pe/ bitstream/handle/UNCP/3920/Vargas $\% 20$ Tupacyupanqui-Montalvo $\% 20$ Morales. pdf?sequence $=1$ \&isAllowed $=y$

Villegas, A. (2017). Resiliencia educativa. ii congreso internacional virtual sobre la educación en el siglo xx. https://www.eumed.net/librosgratis/actas/2017/educacion/59-resilienciaeducativa.pdf

Wu, Z. y McGoogan JM. (2020) Characteristics of and Important Lessons From the Coronavirus Disease 2019 (COVID-19) Outbreak in China. JAMA. 2020;323(13):1239-1242. doi:10.1001/ jama.2020.2648 